\title{
Calidad de vida y de trabajo de los profesores del Instituto Técnico Guaimaral Sede A
}

Mawency Vergel Ortega, José Joaquín Martínez Lozano, Fernel Cárdenas ${ }^{1}$

\section{Resumen}

El presente estudio analiza la calidad de vida de docentes del Instituto Técnico Guaimaral Sede A. Se realizó una investigación analítico-descriptiva, de campo y aplicada, con una población conformada por 23 docentes, describiendo las características actuales de calidad de vida. Se aplica instrumento encuesta, cuestionario estructurado y se realizan talleres participativos para determinar las características actuales de calidad de vida. Se calculó el Índice de Desarrollo Humano (IDH) y sus componentes, describiendo la visión de futuro colectivo de calidad de vida de los docentes, e identificando posibles brechas entre calidad de vida actual y visión de futuro de calidad de vida. Se adaptaron los modelos Mercader y CEPET, para la propuesta. Los resultados indican un IDH alto.

Palabras claves: Calidad de Vida, Docente, futuro.

\section{Abstract}

This study analyzed quality of life of teachers Guaimaral Technical Institute host A. Descriptive analytical and applied field research was conducted with a population consisting of 23 teachers, describing the current characteristics of quality of life. Survey instrument applies; questionnaire and participatory workshops were conducted to determine the current characteristics of quality of life. The Human Development Index (HDI) and its components were calculated, describing the collective vision of future quality of life of teachers, and identifying possible gaps between current quality of life and vision quality of life. Mercader and cepet the models were adapted to the proposal. The results indicate a high HDI.

Keywords: Quality of Life, Teaching, future

1 mawency@ufps.edu.co,jjmartiloz@hotmail.com,fmcg150586hotmail.com

Profesores- Grupo de investigación EULER, ARQUÍMEDES. Universidad Francisco de Paula Santander 


\section{Introducción}

S egún Mercader (1998), la calidad de vida es susceptible de evaluar y se torna más fácil de hacerlo en la medida en que los seres humanos incrementan la capacidad de confiar en su prójimo. Así mismo define la calidad de vida como "el estado de bienestar externo e interno del ser humano", la cual solo es posible de mejorar con la práctica de las virtudes, dado que las mismas, comprimen a parámetros viables las necesidades. Por otra parte, para poder incrementar la calidad de vida, se requiere de acrecentar la educación, porque de hecho se aumentan los niveles de productividad per cápita; además, es función de la actitud asumida frente a las distintas situaciones, por tanto, siempre existirá un agregado de la calidad de vida si se intenta hacer y resolver, analizar y probar diferentes alternativas (p 293,294).

Mercader (1998), también afirma que la calidad de vida está dada por los "diferentes niveles de satisfacción en relación a las necesidades humanas" en cuanto a lo material o físico, psíquico o emocional y lo espiritual; mientras que la calidad de trabajo está determinada por factores como el agrado que se siente en el trabajo, el entusiasmo que se tiene para producir en el mismo y el aprendizaje y superación que se requiere en el trabajo. No obstante, cada persona tiene gustos, habilidades y tendencias diferentes; de hecho, esta circunstancia hace que los porcentajes de contribución e importancia de cada aspecto varíe de una persona a otra en las mismas condiciones de ingreso y de trabajo (p 295, 296).

Con base en lo anterior, el estudio de caso consistió en evaluar la calidad de vida, calidad de trabajo y calidad de vida integral o total en una muestra de profesores del Instituto Técnico Guaimaral Sede A, utilizando las fórmulas de Mercader.

\section{Materiales y Métodos}

La investigación realizada fue de tipo descriptivo, cristalizada en un estudio de caso; el cual se realizó con la intención de analizar y determinar la calidad de vida y de trabajo de un grupo de profesores de una sede y jornada de trabajo del Instituto Técnico Guaimaral.

La información recabada se obtuvo como resultado de la aplicación de un instrumento diseñado por los investigadores, con base en la técnica desarrollada por Mercader. Una vez obtenida la información, se procedió a procesar la misma en una hoja de cálculo, empleando las siguientes fórmulas:

\section{CALIDAD DE VIDA: (CV)}

Material: $n\left(\sum S_{N B}\right)+n\left(\sum S_{N A}\right)+n\left(\sum S_{E G O}\right)$

Psíquico: $n\left(\sum S_{a p}\right)+n\left(\sum S_{e q_{p-e}}\right)$

Espiritual: $n\left(\sum S_{a e}\right)+n\left(\sum S_{C_{s m}}\right)$

CALIDAD DE TRABAJO: (CT)

$n\left(\sum S_{a t}\right)+n\left(\sum S_{e p}\right)+n\left(\sum S_{a s}\right)$

CALIDAD DE VIDA INTEGRAL O TOTAL

$$
C V T=\sqrt{(C V)^{2}+(C T)^{2}}
$$

Las variables medidas e involucradas en el estudio, se relacionan a continuación.

$\mathrm{S}=$ Satisfacción

$\mathrm{NB}=$ Necesidades Básicas

$\mathrm{NA}=$ Necesidades Alternas

ap $=$ Aprendizaje

$\mathrm{eq}_{\mathrm{p}-\mathrm{e}}=$ Equilibrio Psíquico - emocional

ae $=$ Armonía Espiritual

$\mathrm{C}_{\mathrm{sm}}=$ Conocimiento de sí mismo

at $=$ Agrado de trabajo

ep $=$ Entusiasmo de producir

as $=$ Aprendizaje y Superación

$\mathrm{n}=$ Factor de Valoración (Escala 1 - 3)

Autocalificación: (Escala 1-10) 


\section{Resultados y Discusión}

Los profesores del Instituto Técnico Guaimaral tienen características similares en ingreso, nivel educativo, edad y experiencia laboral.
La tabla 1 contiene la data colectada mediante el instrumento utilizado, según lo planteado por Mercader. A cada docente de la muestra se pidió que asignara la importancia a cada factor y que una vez declarado este valor, calificara el respectivo factor en su vida personal y de trabajo.

Tabla 1. Data colectada con el instrumento aplicado.

\begin{tabular}{|c|c|c|c|c|c|c|c|c|c|c|c|c|c|c|c|c|c|c|}
\hline \multirow{2}{*}{ FACTORES DE VALORACIÓN } & \multicolumn{2}{|c|}{ PERS A } & \multicolumn{2}{|c|}{ PERS B } & \multicolumn{2}{|c|}{ PERS C } & \multicolumn{2}{|c|}{ PERS D } & \multicolumn{2}{|c|}{ PERS E } & \multicolumn{2}{|c|}{ PERS F } & \multicolumn{2}{|c|}{ PERS G } & \multicolumn{2}{|c|}{ PERS H } & \multicolumn{2}{|c|}{ PERS I } \\
\hline & Imp. & Calf. & Imp. & Calf. & Imp. & Calf. & Imp. & Calf. & Imp. & Calf. & Imp. & Calf. & Imp. & Calf. & Imp. & Calf. & Imp. & Calf. \\
\hline Necesidades Básicas (NB) & 3 & 10 & 2 & 6 & 3 & 9 & 3 & 9 & 3 & 8 & 3 & 10 & 3 & 9 & 3 & 8 & 3 & 9 \\
\hline Necesidades Alternas (NA) & 3 & 9 & 2 & 7 & 3 & 9 & 2 & 6 & 2 & 5 & 2 & 6 & 2 & 8 & 2 & 6 & 2 & 8 \\
\hline EGO & 2 & 9 & 2 & 8 & 3 & 8 & 2 & 7 & 2 & 5 & 1 & 4 & 2 & 7 & 2 & 6 & 2 & 8 \\
\hline Necesidades de Aprendizaje (ap) & 3 & 10 & 3 & 7 & 3 & 8 & 3 & 9 & 2 & 7 & 3 & 10 & 3 & 9 & 3 & 8 & 3 & 9 \\
\hline Equilíbrio Psíquico-emocional (EQp-e) & 3 & 9 & 3 & 8 & 3 & 9 & 3 & 9 & 3 & 8 & 2 & 8 & 3 & 9 & 3 & 8 & 3 & 9 \\
\hline Armonía Espiritual (ae) & 3 & 10 & 3 & 8 & 3 & 8 & 2 & 8 & 2 & 6 & 3 & 10 & 3 & 9 & 3 & 9 & 3 & 8 \\
\hline Conocimiento de si mismo (Csm) & 3 & 10 & 3 & 8 & 3 & 8 & 3 & 9 & 3 & 8 & 2 & 8 & 2 & 9 & 3 & 7 & 3 & 8 \\
\hline Agrado de Trabajo (at) & 3 & 10 & 3 & 9 & 3 & 9 & 2 & 9 & 3 & 8 & 3 & 10 & 3 & 9 & 3 & 8 & 3 & 9 \\
\hline Entusiasmo de Producir (ep) & 3 & 9 & 3 & 9 & 3 & 10 & 3 & 9 & 3 & 9 & 3 & 10 & 3 & 9 & 3 & 7 & 3 & 8 \\
\hline Aprendizaje y Superación (as) & 3 & 10 & 3 & 9 & 3 & 8 & 3 & 9 & 3 & 10 & 3 & 10 & 3 & 9 & 3 & 7 & 3 & 9 \\
\hline
\end{tabular}

Fuente: instrumento aplicado por autores

En la tabla 2 aparecen los cálculos realizados para cada factor, resultado de multiplicar la Importancia asignada al factor y la calificación del mismo, por cada una de los docentes.

Tabla 2. Resultado individual por factor en cada uno de los docentes de la muestra.

\section{FACTORES}

Necesidades Básicas (NB)

Necesidades Alternas (NA)

EGO

Necesidades de Aprendizaje (ap)

Equilíbrio Psíquico-emocional (EQp-e)

Armonía Espiritual (ae)

Conocimiento de si mismo (Csm)

Agrado de Trabajo (at)

Entusiasmo de Producir (ep)

Aprendizaje y Superación (as)

$\begin{array}{ccccccccc}\text { PERS } & \text { PERS } & \text { PERS } & \text { PERS } & \text { PERS } & \text { PERS } & \text { PERS } & \text { PERS } & \text { PERS } \\ \text { A } & \text { B } & \text { C } & \text { D } & \text { E } & \text { F } & \text { G } & \text { H } & \text { I } \\ 30 & 12 & 27 & 27 & 24 & 30 & 27 & 24 & 27 \\ 27 & 14 & 27 & 12 & 10 & 12 & 16 & 12 & 16 \\ 18 & 16 & 24 & 14 & 10 & 4 & 14 & 12 & 16 \\ 30 & 21 & 24 & 27 & 14 & 30 & 27 & 24 & 27 \\ 27 & 24 & 27 & 27 & 24 & 16 & 27 & 24 & 27 \\ 30 & 24 & 24 & 16 & 12 & 30 & 27 & 27 & 24 \\ 30 & 24 & 24 & 27 & 24 & 16 & 18 & 21 & 24 \\ 30 & 27 & 27 & 18 & 24 & 30 & 27 & 24 & 27 \\ 27 & 27 & 30 & 27 & 27 & 30 & 27 & 21 & 24 \\ 30 & 27 & 24 & 27 & 30 & 30 & 27 & 21 & 27\end{array}$

Fuente: cálculos realizados por autores con base en las fórmulas de Mercader 
Tabla3. Calidad de vida, calidad de trabajo y calidad de vida integral o total.

\section{RESULTADOS CALIDAD DE VIDA}

$\begin{array}{lccccccccc}\text { PERSONA } & \text { A } & \text { B } & \text { C } & \text { D } & \text { E } & \text { F } & \text { G } & \text { H } & \text { I } \\ \text { MATERIAL / FÍSICA } & 75 & 42 & 78 & 53 & 44 & 46 & 57 & 48 & 59 \\ \text { PSÍQUICAS / EMOCIONALES } & 57 & 45 & 51 & 54 & 38 & 46 & 54 & 48 & 54 \\ \text { ESPIRITUAL } & 60 & 48 & 48 & 43 & 36 & 46 & 45 & 48 & 48 \\ \text { CALIDAD DE VIDA } & 192 & 135 & 177 & 150 & 118 & 138 & 156 & 144 & 161 \\ \text { CALIDAD DE TRABAJO } & 87 & 81 & 81 & 72 & 81 & 90 & 81 & 66 & 78 \\ \begin{array}{l}\text { CALIDAD DE VIDA INTEGRAL O } \\ \text { TOTAL }\end{array} & & & & & & & & & \\ & 210,8 & 157,4 & 194,7 & 166,4 & 143,1 & 164,8 & 175,8 & 158,4 & 178,9\end{array}$

Fuente: cálculos realizados por autores con base en las fórmulas de Mercader.

Se encontró que, no siempre los docentes con valores altos en calidad de vida, también poseen valores altos en calidad de trabajo, como se puede apreciar en la tabla 3 los docentes B, E y F presentan los valores más bajos en calidad de vida, pero la calidad en el trabajo es mayor a otros con valores de calidad vida superiores a los mencionados anteriormente, lo que hace pensar que estas personas son felices en su trabajo aunque no se sientan $\tan$ felices supliendo sus necesidades físicas, emocionales y espirituales.

Así mismo la calidad de vida integral de los docentes B, E y F, no dista mucho de los demás docentes, por tanto se puede concebir que en general los docentes del Instituto guardan equilibrio entre la calidad de vida y la calidad de trabajo y que de hecho esos valores son altos, por tanto, se puede considerar que dichos docentes son felices en su trabajo y en su vida.

Con respecto a la diferencia por estado civil, se encontró en general que los docentes solteros (A, C y D) integralmente son más felices que los docentes casados y con respecto al género las mujeres $(A, C, G, I)$ son más felices que los hombres.

La escala de valores de IDH se subdivide en tres categorías: a) desarrollo humano alto, con valores entre 0.800 a 1, b) desarrollo humano medio, con valores entre 0,500 y 0,799 , c) desarrollo humano bajo, con valores entre 0 a 0,499, inclusive.

Con base a estas consideraciones se determinó el IDH para la los docentes del Instituto, obteniéndose un valor de 0,894 que corresponde a la ca- tegoría de desarrollo humano alto. Se puede observar como el componente ingreso condiciona fuertemente el valor de IDH, seguido por el componente logro educativo; igualmente la principal actividad económica es la docencia, caracterizada por tener alto conocimiento de tecnologías, pedagogía, emprenderismo, educación a jóvenes con discapacidad, lo cual redunda en satisfacción, amor por la profesión y la labor social, lo que origina un índice de desarrollo humano alto en los docentes del Instituto Técnico Guaimaral.

La visión de futuro colectiva de calidad de vida en la institución, de los docentes, en su mayoría corresponde a un futuro inmediato (1 año); es decir, la visión que tienen sobre el desarrollo de su comunidad es limitada al primer año, porque esperan resolver problemas en el corto plazo, ya que esta realidad les impide abordar metas más complejas, tal y como lo significa Maslow citado por Ramakrishna.

Asimismo, estos autores afirman que es una gran necesidad establecer una comunicación noble y promover relaciones armónicas entre los miembros de la comunidad y que la organización empresarial es una vía para la promoción de la mejora social entre otras.

No obstante, se encuentra orgullo y satisfacción por los logros colectivos alcanzados, el reconocimiento de la comunidad, la organización de los procesos y alta satisfacción por certificación de la Institución con Normas ISO 90012008 y NTCGP 10002009. 
Plan de trabajo. Los principales problemas que podrían limitar un futuro desarrollo de la comunidad, producto del taller participativo de investigación comunitaria, fueron los siguientes: a) pocos recursos económicos, b) comunicación c) roles acorde a formación profesional d) desconocimiento de nuevas tecnologías, redes sociales, tablets $\mathrm{u}$ otros en procesos de aprendizaje f) inadecuada figura organizativa, j) poca participación comunitaria.

Los lineamientos estratégicos o guías de acción que permitirán diseñar los programas requeridos en cada una de las áreas críticas, detectadas a través de los diferentes diagnósticos realizados (diagnósticos participativos y cuestionarios), y que a su vez facilitarán el alcance de la visión colectiva del Instituto Técnico Guaimaral son: el fortalecimiento organizacional, continuar implementación y certificación de calidad de la institución y la capacitación.

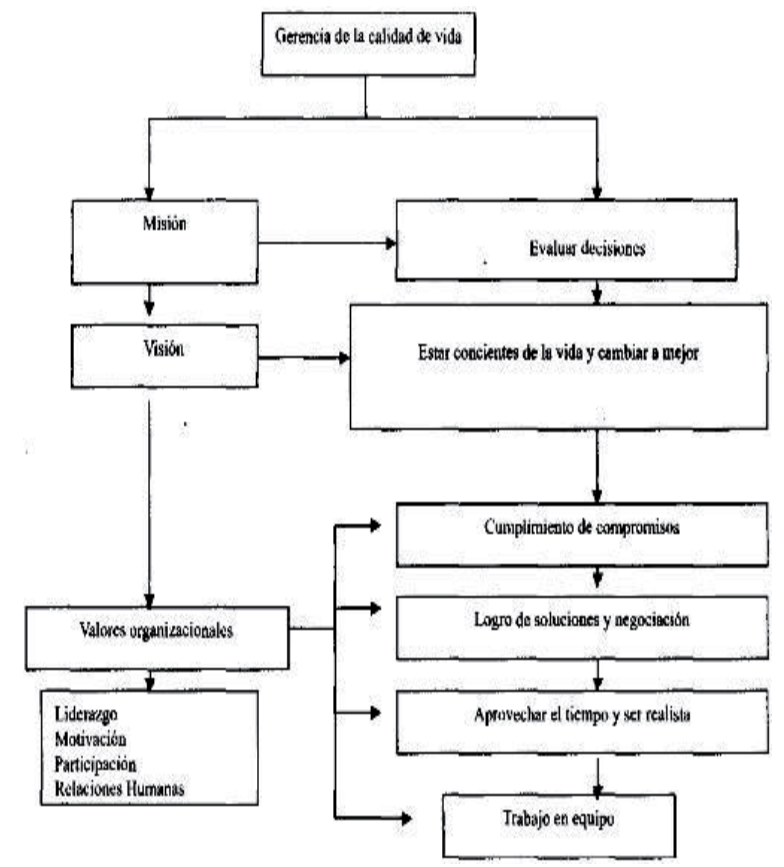

Fuente: Adaptado de Mercader (1998) y del CEPET (1997)

\section{Conclusiones}

Los docentes del Instituto Técnico Guaimaral Sede A, guardan equilibrio entre la calidad de vida y la calidad de trabajo, se puede considerar que son felices en su trabajo y en su vida.

La visión colectiva de calidad de vida es de gran proyección, donde los docentes vislumbran amplias capacidades para abordar realidades internas y externas, cuando interactúan, solidariamente, bajo un esquema de valores.

Los docentes solteros son más felices que los docentes casados y con respecto al género, las mujeres son más felices que los hombres.

El IDH para la los docentes del Instituto corresponde a la categoría de desarrollo humano alto. Y un plan estratégico contemplaría ejes centrados en valores organizacionales, la capacitación, continuar la implementación de nuevas tecnologías en el aula, continuar procesos de certificación de alta calidad, y fortalecimiento de roles y procesos de comunicación.

\section{Bibliografía}

Mercader, V. 1998. Gerencia de la Vida. Editado por Víctor Mercader. Caracas Venezuela. ISBN 980-07-2261-0

Mercader, V. 1998. Gerencia versus Calidad de Vida. Edición especial del Congreso de RRHH. Revista ANRI. Caracas, Venezuela.

Pérez, J. J. 2000. La Planificación participativa aplicada al diseño de un programa de extensión agrícola dirigido a productores de la comunidad La Estrella. La universidad del Zulia. Facultad de Agronomía. División de estudios para graduados. Programa gerencia de agrosistemas (Trabajo de grado). Maracaibo, Venezuela. xi, 78 pp

Ramakrishna, B. 1992. Comunicación y desarrollo rural. Espasante, SRL. Caracas. $52 \mathrm{p}$

SAS Institute, Inc. 1990. User's Guide. Version 6. $4^{\circ}$ edition. SAS Inst. Inc., Cary, NC. USA

Serna, H. 1996. Planeación y gestión estratégica. Serie Gerentes. RAM Editores. 4ta Edición. Colombia-pp:30-34

Torres, G. 2000. Un sueño para Venezuela. Banco Venezolano de Crédito. 2da. Edición. Venezuela. $157 \mathrm{pp}$.

Vallejo, C. 1992. El municipio como escenario del desarrollo económico y social. Cuadernos de agroindustria y economía rural. № 28. Colombia, p. 19-38. 